\title{
Chemotherapy alters ancestry relapse-risk differences in childhood ALL
}

While the cure rates for children with childhood acute lymphoblastic leukemia (ALL) are high, some children are not cured and many do not benefit from existing therapies owing to unacceptable adverse effects. Many clinical studies have shown that survival outcomes vary according to ethnic differences, with poor survival observed among children of African American origin or Hispanic ancestry. The causes of these different outcomes are not clear but both genetic and non-genetic factors are important. Now, an article published in Nature Genetics by Jun Yang and coauthors has not only pinpointed the germline polymorphisms associated with the variation in outcomes but has also shown that ancestry-related differences in risk of relapse were overcome by an extra phase of chemotherapy.

"We undertook a series of studies to elucidate the germline polymorphisms associated with variation in drug response in childhood ALL," explains Mary Relling, senior investigator of the study. She continues, "we also wanted to use a genome-wide approach to find associations between single nucleotide polymorphism (SNP) genotypes and response in order to obviate the biases inherent in candidate gene approaches". The researchers assessed the germline SNP genotypes in an unselected large cohort of children with ALL and tested if genetic ancestry was associated with treatment outcome. The cumulative incidence of relapse risk was significantly associated with Native American ancestry, which was highest among Hispanics. "These findings are consistent with prior work showing that Hispanic children with ALL tend to have a higher relapse rate than non-Hispanic whites, and strongly suggest a genomic component to their increased relapse risk".

Early response to therapy in ALL is an important indicator of relapse risk, and this is determined by the level of minimal residual disease after induction therapy. Even in patients who had a low minimal residual disease risk, Native American ancestry was associated with a higher risk of relapse. This finding has important clinical implications as the ability to identify a subgroup of patients who may need more-intensive therapy despite no minimal residual disease would aid in prognostication.

In patients with a high Native American ancestry, the risk of relapse was highest in children who did not receive delayed intensification chemotherapy; however, this outcome was not observed in children who received such therapy. As Relling highlights, "Importantly, the poor prognosis associated with Native American ancestry disappeared among those who were randomly assigned to receive an extra phrase of chemotherapy." The investigators comment that the benefits of delayed intensification are likely to outweigh the cost in patients with $>10 \%$ Native American ancestry.

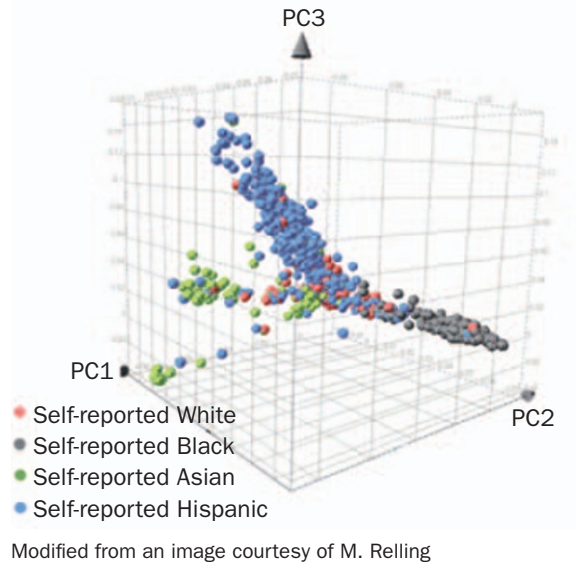

The investigators were interested to explore the mechanisms by which ancestry affected risk of relapse. Jun Yang, primary author of the study, showed that a SNP in the gene $P D E 4 B$ had the greatest risk associated with hematologic relapse. Primary ALL cells expressing PDE4B were more resistant to prednisolone, indicating this gene might have a role in response. In the future, Yang's team plan to "study the specific genes and mechanisms that confer the ancestry-related poor prognosis, to understand better which chemotherapy agents to use to maximize response for different patients."

\section{Lisa Hutchinson}

Original article Yang, J. J. et al. Ancestry and pharmacogenomics of relapse in acute lymphoblastic leukemia. Nat. Genet. 43, 237-241 (2011) 